\title{
Single Stage Dual Active Bridge AC-DC Converter with Active Power Decoupling
}

\author{
Davide Gottardo, Liliana De Lillo, Lee Empringham, Alessandro Costabeber, Linglin Chen \\ PEMC Research Group \\ Department of Electrical and Electronic Engineering \\ University of Nottingham \\ Email: davide.gottardo@nottingham.ac.uk
}

\begin{abstract}
The paper introduces a new a isolated DCAC DAB converter topology, capable of active power decoupling on the $\mathrm{AC}$ side of the transformer, made up of two full bridges with no additional active component. The differential voltage modulated by the DC-side full bridge is used to control the voltage applied to the primary winding of a high-frequency transformer, while the common mode voltage generated by the $\mathrm{AC}$-side full bridge is applied to the secondary windings.

The choice of applying the common mode voltage ripple of the $\mathrm{AC}$-side full bridge to the transformer, allows to have the required ripple voltage to transfer the power, while keeping the differential voltage ripple, that is applied to the AC output, at its minimum value, making possible the use of a small AC filter inductor.

A control strategy is proposed and validated by simulation.

This work is being developed at University of Nottingham within the framework of project SENSIBLE.

Index Terms-Active power decoupling, single-phase system, dual active bridge, DAB, AC-DC DAB.
\end{abstract}

\section{INTRODUCTION}

When galvanic isolation is required, the dual active bridge (DAB) topology is widely used, because of its high efficiency and power density [1], [2]. For this reason, many AC-DC converters that require galvanic insulation are made by two stages: an isolated DC-DC DAB stage followed by a VSI or CSI DC-AC inverter [3]. In case of unidirectional power transfer from $\mathrm{DC}$ to $\mathrm{AC}$, such as in $\mathrm{PV}$ generation, the $\mathrm{AC}$ side bridge can be a simple diode rectifier, otherwise, a full bridge is used.

In case of single phase AC systems, one or more power decoupling elements have to be included in the converter, in order to deal with the ripple power components [4], [5]. This power decoupling action can be provided on the $\mathrm{AC}$ side of the isolation transformer, or on the DC side. If the power decoupling action is performed on the $\mathrm{AC}$ side, the power exchanged through the transformer is equal to the power exchanged through the DC port of the converter, which is constant, while if power decoupling is performed on the DC side, it is equal to the power exchanged through the $\mathrm{AC}$ port, that oscillates between zero and double DC power at twice grid frequency. For this reason, ACside power decoupling is usually better in terms of efficiency of the transformer and its driving circuit.

In order to increase the efficiency and/or reduce the number of active devices, single stage AC-DC DAB are used [6]-[9]. However, in the topologies considered in [8], [9], the ripple current coming from the transformer is present on the AC modulated output of the converter, and has to be filtered out by adequate passive filters, while in [6], [7] a reverse blocking capable $\mathrm{H}$ bridge is required, with a total of 8 active switches on the AC side, the same number required in a two stage converter. In [7] active power decoupling is achieved on the DC side, while in [8], [9] power decoupling is not provided.

The proposed topology is a single stage ACDC dual active bridge, that provides active power decoupling on the AC side of the converter. The topology has a total of 4 active switches on the $\mathrm{AC}$ side, and the ripple current flowing through the transformer does not appear on the AC modulated output. 


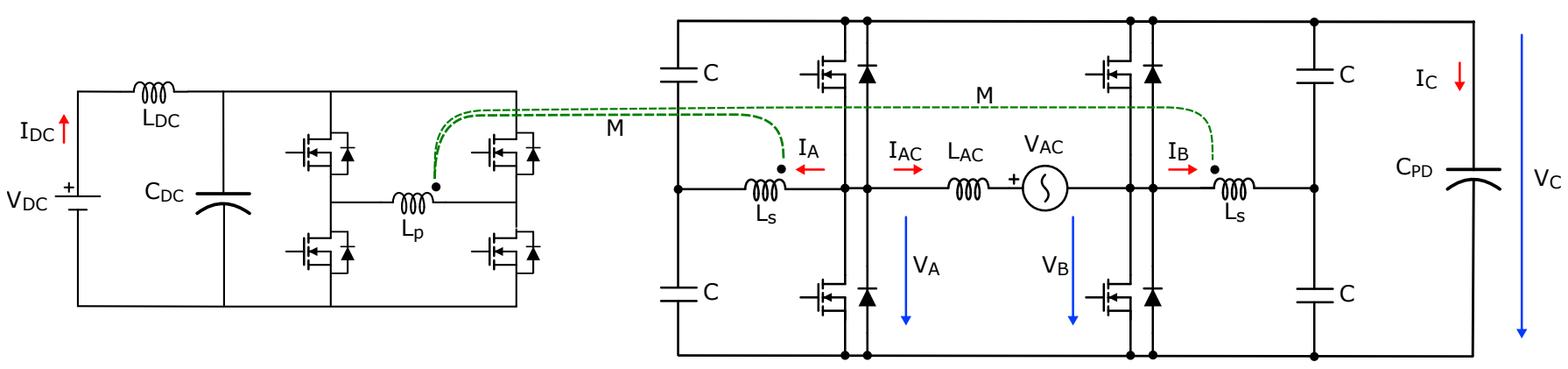

Fig. 1: Proposed topology
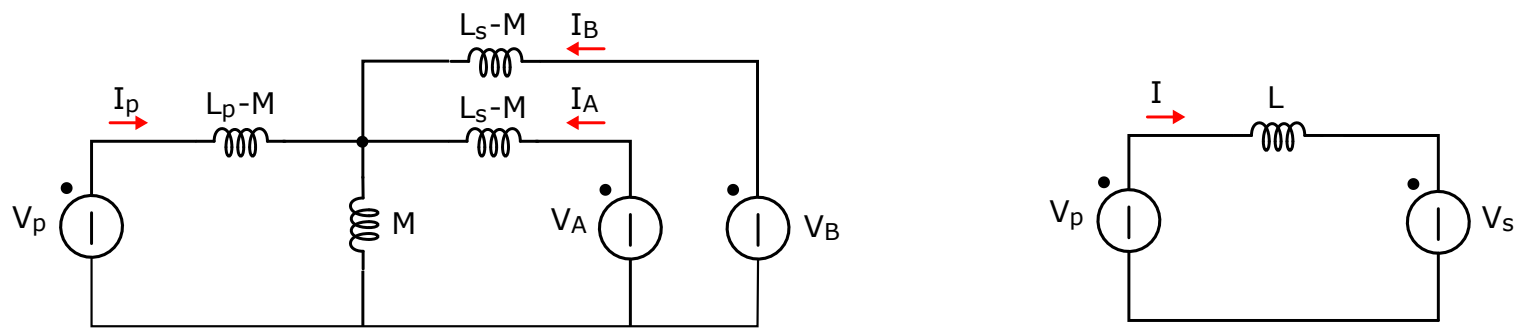

(a) Primary equivalent circuit

(b) $V s=\left(V_{A}+V_{B}\right) / 2, L=L p-M+(L s-M) / 2$

Fig. 2: High frequency equivalent circuit

\section{PRoposed TOPOLOGY}

The proposed topology, shown in figure 1, is constituted by two $\mathrm{H}$ bridges, a high frequency transformer with one primary and two equal secondary windings, a power decoupling capacitor $C_{P D}$, an $\mathrm{AC}$ filter inductor $L_{A C}$, and four DC blocking capacitors $C$. Those capacitors are sized in order to block the flow of DC or low-frequency currents across the transformer windings, while behaving like a closed circuit for the ripple components at switching frequency. Since those capacitors are subjected to high current ripple, low ESR is required.

The differential voltage modulated by the ACside $\mathrm{H}$ bridge is used to control the $\mathrm{AC}$ current, while the modulated outputs of its two legs are connected to one end of each of the transformer secondary windings, closing the circuit through the DC blocking capacitors. This has the equivalent effect of applying the common mode voltage ripple of the $\mathrm{AC}$ side $\mathrm{H}$ bridge to a single secondary winding of the high voltage transformer. The DC blocking capacitors also contribute to lower the ripple current flowing through $C_{P D}$, thus reducing its $I^{2} t$ stress.
TABLE I: Circuit parameters

\begin{tabular}{lrclrc}
\hline Name & Value & Unit & Name & Value & Unit \\
\hline$V_{G}$ & 230 & $V_{R M S}$ & $V_{D C}$ & 500 & $V$ \\
$C_{P D}$ & 55 & $\mu F$ & $C$ & 0.1 & $\mu F$ \\
$L_{A C}$ & 0.5 & $m H$ & $M$ & 600 & $\mu H$ \\
$L p$ & 666 & $\mu H$ & $L s$ & 666 & $\mu H$ \\
$L_{D C}$ & 10 & $\mu H$ & $C_{D C}$ & 10 & $\mu F$ \\
\hline
\end{tabular}

The DC-side $\mathrm{H}$ bridge is used to drive the primary side of the transformer, in order to transfer the required power. Power decoupling is performed by capacitor $C_{P D}$ on the $\mathrm{AC}$-side, so that the power transferred by the transformer is continuous across the entire AC period. Since the voltage ripple applied on $C_{P D}$ can be high, a small capacitance is required, enabling the use of reliable film capacitors.

The AC-side half bridge legs are modulated in phase, with a steady-state duty cycle equal to $0.5 \pm$ $\frac{V_{A C}}{2 V_{C}}$, so that the differential voltage is equal to $V_{A C}$.

\section{CONTROL STRUCTURE}

The control system is formed by three control loops: the AC current controller, the DC power controller and the power decoupling capacitor DC 


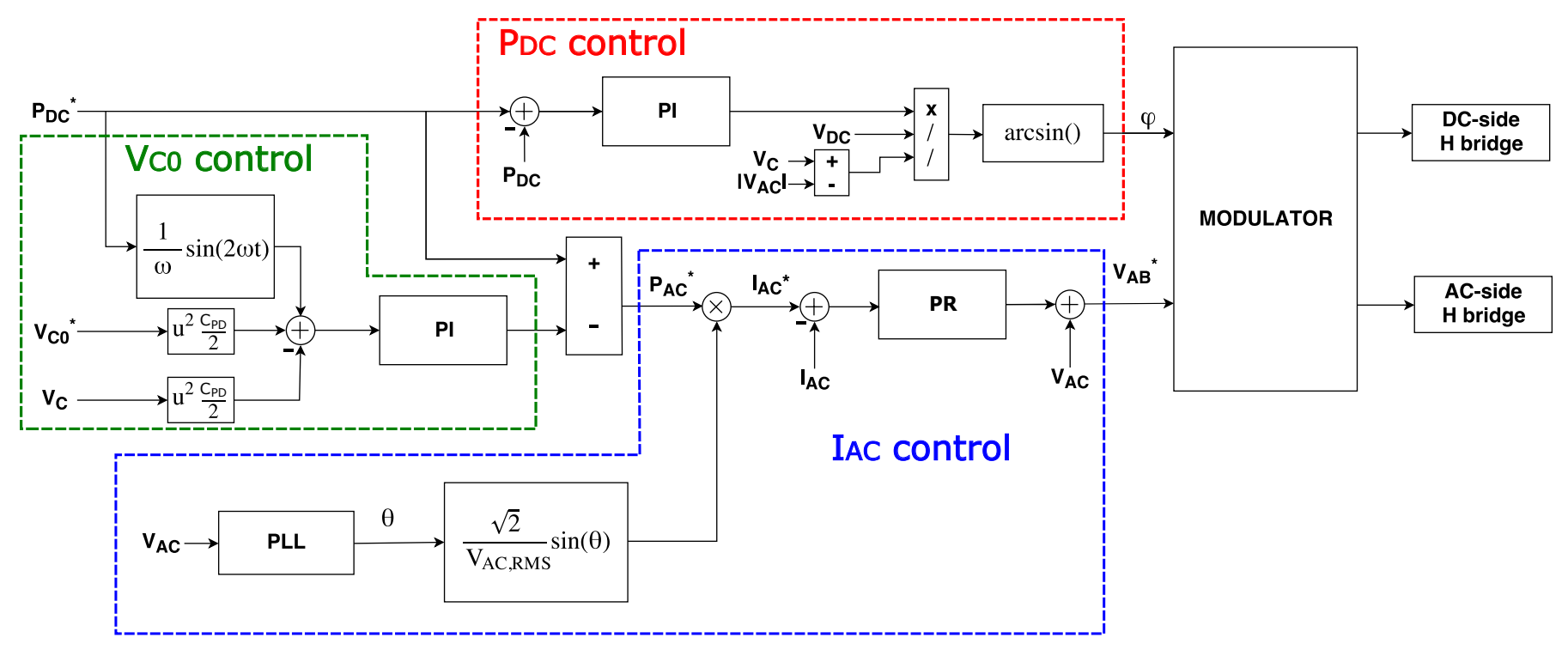

Fig. 3: Control structure

voltage offset controller. The overall control structure is shown in figure 3 .

In order to control the system, five measurements are taken:

- DC voltage and current $V_{D C}$ and $I_{D C}$;

- AC voltage and current $V_{A C}$ and $I_{A C}$;

- power decoupling capacitor voltage $V_{C}$.

\section{A. DC power}

The DC power controller regulates the DC power by acting on the outer phase shift $\varphi$.

Assuming to consider only the fundamental component of the voltages applied to the primary and the secondary of the transformer, the power transfer is estimated as:

$$
P \simeq \frac{V_{p} V_{s}}{2 \pi f_{S W} L} \sin (\varphi)
$$

where $f_{S W}$ is the switching frequency, $L$ is the leakage inductor of the transformer, $\varphi$ is the phase shift between the two voltages, and $V_{p}$ and $V_{s}$ are the amplitudes of the fundamental components at switching frequency of the voltages applied to the primary and secondary of the transformer, as shown in figure $2 \mathrm{~b}$.

As $V_{s}$ is the common mode voltage modulated by the AC full bridge, its amplitude and waveform change during the grid period. Approximately, the amplitude of the fundamental component of $V_{s}$ is proportional to the difference between the DC-side of the full bridge, which is $V_{C}$, and the differential voltage modulated by the bridge, which is $V_{A C}$. In the same way, the fundamental component of $V_{p}$ is proportional to $V_{D C}$. Thus:

$$
\begin{aligned}
& V_{s} \propto V_{C}-\left|V_{A C}\right| \\
& V_{p} \propto V_{D C}
\end{aligned}
$$

Substituting in eq.1:

$$
P \propto V_{D C}\left(V_{C}-\left|V_{A C}\right|\right) \sin (\varphi)
$$

Inverting this equation allows to linearise the control loop that regulates the power, so that in steady state a constant power reference corresponds to a constant output of the controller.

\section{B. Power decoupling capacitor offset voltage}

The difference between the instantaneous power flowing in the $\mathrm{AC}$ port $P_{A C}$ and the power coming from the DC side $P$ is stored in the capacitor $C_{P D}$. Assuming an ideal converter (zero power loss, constant $P$ and perfectly sinusoidal $V_{A C}$ and $I_{A C}$ ), the integral of $P-P_{A C}$, that is the energy ripple on $C_{P D}$, is equal to [10]:

$$
E_{R}=\frac{P}{\omega} \sin (2 \omega t)
$$

Since the mean value over the AC period of $E_{R}$ is zero, it involves a ripple voltage but does not affect the DC offset voltage on the capacitor. In general, the mean value of $P-P_{A C}$ is non-zero, and that 

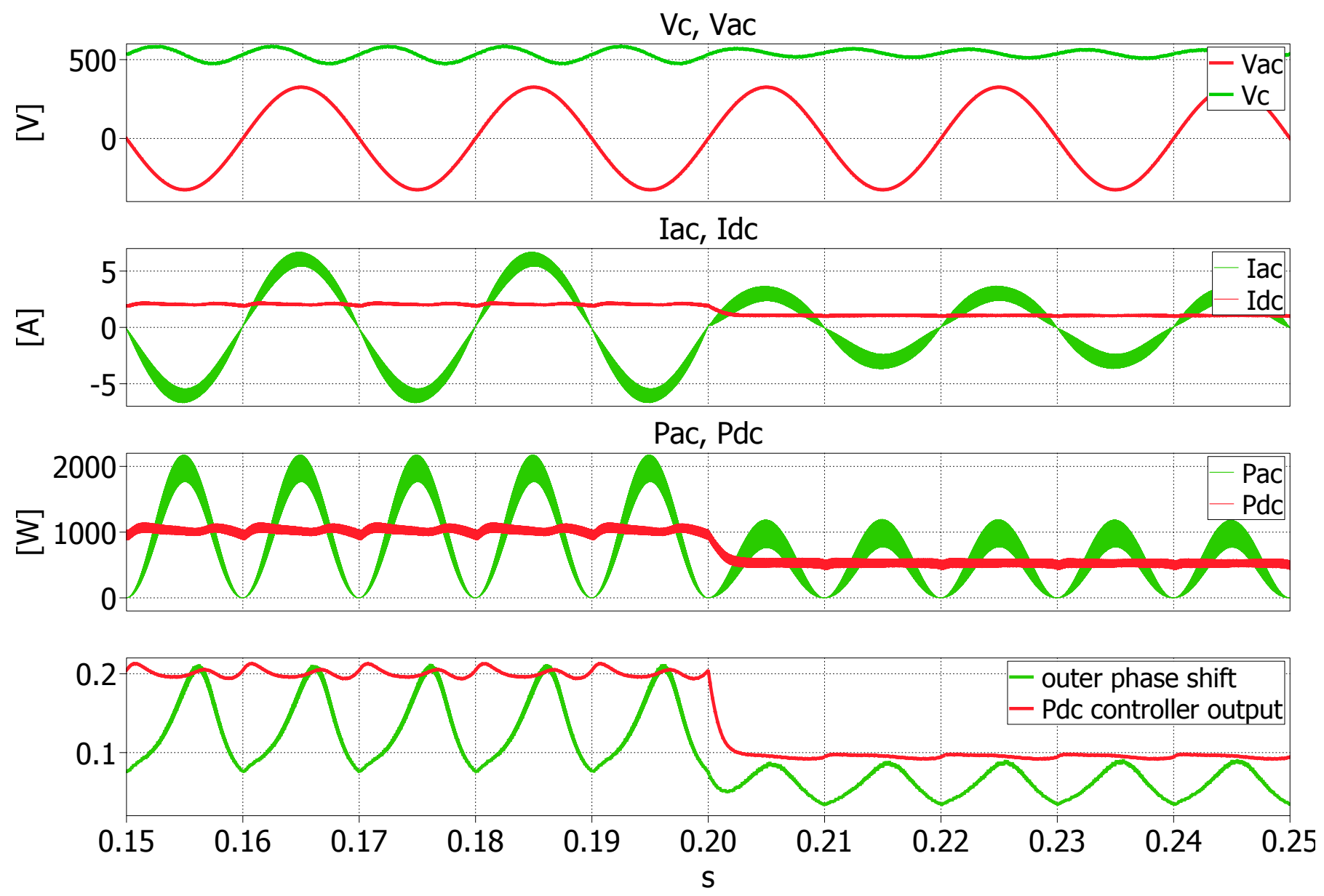

Fig. 4: Simulation results: step power transfer from $1 \mathrm{~kW}$ to $0.5 \mathrm{~kW}$ at $\mathrm{t}=0.2 \mathrm{~s}$

translates in a change of the offset energy on the capacitor, which is approximately proportional to the square of its DC voltage offset.

The capacitor offset voltage controller changes the AC power set-point in order to control the average energy stored in the power decoupling capacitor $C_{P D}$, while maintaining a constant DC power. In order to remove the $100 \mathrm{~Hz}$ ripple, a notch filter is generally included in the controller [5]. In this case, instead, the ideal ripple energy, shown in eq.5, is calculated and cancelled out, so that the input of the controller is the mean stored energy error. In steady-state, the output of the controller is equal to the average power losses of the converter, plus a very small ripple.

\section{AC current}

The AC current controller takes the reference current calculated starting from the reference $\mathrm{AC}$ power, and outputs the desired differential voltage
$V_{A B}$ to be modulated by the AC-side full bridge. A proportional-resonant controller tuned at $50 \mathrm{~Hz}$ was chosen.

Simple PI controllers were used for all the other control loops, achieving good simulation results.

\section{Simulations}

In order to validate the proposed control structure, simulations were made using Simulink and the PLECS Blockset simulation tool.

The circuit parameters used are shown in table I. The chosen switching frequency $f_{S W}$ is $100 \mathrm{kHz}$.

Figure 4 shows the behaviour of the converter during a transient from $1 \mathrm{~kW}$ to $0.5 \mathrm{~kW}$.

\section{CONCLUSION}

The paper introduces a new topology for a isolated DC-AC DAB converter, capable of active power decoupling on the $\mathrm{AC}$ side of the transformer, 
made up of two full bridges with no additional active component. The differential voltage modulated by the DC-side full bridge is used to control the voltage applied to the primary winding of a highfrequency transformer, while the common mode voltage generated by the $\mathrm{AC}$-side full bridge is applied to the secondary windings.

The choice of applying the common mode voltage ripple of the AC-side full bridge to the transformer allows to have enough ripple voltage to transfer the power, while keeping the differential voltage ripple at its minimum value, making possible the use of a small AC filter inductor.

The simulation results confirm the correct behaviour of the converter and of the control system.

The limited number of active and the small size of the required passive components contribute to make this topology an interesting solution for application such as PV distributed generation and energy storage solutions.

\section{ACKNOWLEDGMENT}

This project has received funding from the European Unions Horizon 2020 research and innovation programme under grant agreement No 645963.

\section{REFERENCES}

[1] W. A. A. D. Doncker and D. M. Divan, "A Three-phase SoftSwitched Applications / I = q," IEEE Transactions on Industry Applications, vol. 27, no. 1, 1991.

[2] F. Krismer and J. W. Kolar, "Efficiency-Optimized HighCurrent Dual Active Bridge Converter for Automotive Applications," IEEE Transactions on Industrial Electronics, vol. 59, no. 7, pp. 2745-2760, 2012.

[3] J. K. Pedersen, S. B. Kjaer, J. K. Pedersen, S. Member, and F. Blaabjerg, "A Review of Single-Phase Grid-Connected Inverters for Photovoltaic Modules A Review of Single-Phase Grid-Connected Inverters for Photovoltaic Modules," IEEE TRANSACTIONS ON INDUSTRY APPLICATIONS, VOL. 41, NO. 5, SEPTEMBER/OCTOBER 2005, vol. 41, no. OCTOBER, pp. 1292-1306, 2005.

[4] P. T. Krein, R. S. Balog, and M. Mirjafari, "Minimum Energy and Capacitance Requirements for Single-Phase Inverters and Rectifiers Using a Ripple Port," IEEE Transactions on Power Electronics, vol. 27, pp. 4690-4698, nov 2012.

[5] Y. Tang, W. Yao, P. C. Loh, and F. Blaabjerg, "Highly Reliable Transformerless Photovoltaic Inverters with Leakage Current and Pulsating Power Elimination," IEEE Transactions on Industrial Electronics, vol. 63, pp. 1016-1026, feb 2016.

[6] K. Vangen, T. Melaa, and A. Adnanes, "Soft-switched highfrequency, high power DC/AC converter with IGBT," PESC '92 Record. 23rd Annual IEEE Power Electronics Specialists Conference, pp. 26-33, 1992.
[7] J. You, D. M. Vilathgamuwa, N. Ghasemi, and W. L. Malan, "LLC circuit based ripple current suppression method for single phase bidirectional DC-AC DAB converter," 2017 IEEE 3rd International Future Energy Electronics Conference and ECCE Asia (IFEEC 2017 - ECCE Asia), vol. 1, no. 2, pp. 682-686, 2017.

[8] G. Castelino, K. Basu, N. Weise, and N. Mohan, "A bidirectional, isolated, single-stage, DAB-based AC-DC converter with open-loop power factor correction and other advanced features," 2012 IEEE International Conference on Industrial Technology, ICIT 2012, Proceedings, pp. 938-943, 2012.

[9] F. Jauch and J. Biela, "Combined Phase-Shift and Frequency Modulation of a Dual-Active-Bridge AC-DC Converter with PFC," IEEE Transactions on Power Electronics, vol. 31, no. 12, pp. 8387-8397, 2016.

[10] D. Gottardo, L. D. Lillo, L. Empringham, and A. Costabeber, "Differential buck single phase grid connected ac-dc converter with active power decoupling using a flipping capacitor," in 2017 IEEE 8th International Symposium on Power Electronics for Distributed Generation Systems (PEDG), pp. 1-5, April 2017. 